\section{Pregresse IST}

\begin{tabular}{cccc}
\hline Sì & 32 & $10(32.3 \%)$ & 0.001 \\
\hline No & 286 & $28(9.8 \%)$ & \\
\hline ND & 9 & 5 & \\
\hline Disturbi nel partner & & & \\
\hline Sì & 47 & $12(25.5 \%)$ & 0.001 \\
\hline No & 246 & $22(8.9)$ & \\
\hline ND & 34 & 9 &
\end{tabular}

\section{Conclusioni.}

In accordo con $\mathrm{i}$ dati della letteratura internazionale, $\mathrm{i}$ fattori di rischio da noi individuati sono stati: la giovane età del primo rapporto sessuale, non avere un partner stabile, l'aver avuto uno o più partners occasionali o più di un partner negli ultimi 6 mesi una storia di pregresse IST, la presenza di una sintomatologia genitale nel partner. I dati ad oggi in nostro possesso ci indicano che 221 donne $(67.6 \%)$ hanno almeno un altro fattore di rischio oltre la giovane età ed in questo gruppo la prevalenza dell'infezione da C.t. è del 18.6\%. In 260 casi è stato anche possibile ricercare la presenza di altre IST che sono state diagnosticate in 27 pazienti $(10.4 \%)$.

\section{C03.2}

\section{BACTEROIDES THETAIOTAOMICRON ED INFEZIONE POSTISTERECTOMIA}

\author{
Del Gaudio T.',Tajani E. ${ }^{2}$, Miragliotta G. ${ }^{3}$, \\ Mosca A. ${ }^{3}$
}

'Laboratorio Analisi P.O. di Andria,

¿U.O. Ostetricia e Ginecologia P.O. di Andria, AUSL BAT/I, 7003 I Andria

3Sezione di Microbiologia, Dipart. MIDIM,

Facoltà di Medicina e Chirurgia, Università di Bari,

70124 Bari

\section{Introduzione.}

Le infezioni conseguenti ad isterectomia transvaginale non sono ancora completamente conosciute. Sebbene la flora endogena vaginale rappresenti l'origine dell'infezione per via ascendente, non sono definiti i fattori di rischio che determinano la formazione di ascesso a livello della cupola vaginale, pur in presenza di adeguata risposta dei meccanismi di difesa della paziente.

Metodi.

Viene presentato il caso di una paziente di 44 anni, sottoposta ad isterectomia per utero fibromiomatoso, prolasso uterino ed ipermenorrea, con successiva formazione di ascesso della cupola vaginale. L'indagine microbiologica del materiale purulento raccolto con drenaggio intraoperatoriamente è consistita in esame batterioscopico previa colorazione di Gram, coltivazioni in aerobiosi/anaerobiosi, identificazione median- te Rapid API-32A system (bio-Mérieux, Italia) e conferma con Hewlett-Packard 6890 series gas liquid chromatography system, sensibilità antibiotica con Etest (AB Biodisk, Solna, Sweden). È stato analogamente processato un tampone vaginale eseguito contemporaneamente.

\section{Risultati.}

Tanto dal materiale purulento quanto dal tampone vaginale è stato isolato in coltura pura ed identificato Bacteroides thetaiotaomicron (percentuale di identificazione con Rapid API-32 $99.9 \%$; profilo di similarità all'esame gas-cromatografico 0,608 (prima scelta). B. thetaiotaomicron è risultato beta-lattamasi positivo, sensibile ad Amoxicillina/clavulanato (MIC 0.75 $\mu \mathrm{g} / \mathrm{mL})$, metronidazolo $(2 \mu \mathrm{g} / \mathrm{mL})$, imipenem $(0.25$ $\mu \mathrm{g} / \mathrm{mL})$, moxifloxacin $(1.5 \mu \mathrm{g} / \mathrm{mL})$; resistente ad amoxicillina $(256 \mu \mathrm{g} / \mathrm{mL})$, piperacillina-tazobactam $(256$ $\mu \mathrm{g} / \mathrm{mL})$, e cefoxitin $(256 \mu \mathrm{g} / \mathrm{mL})$.

\section{Conclusioni.}

Le infezioni genitali femminili sono solitamente miste e $B$. thetaiotaomicron è stato raramente isolato $(<1 \%)$. Lo spettro di sensibilità antibiotica è risultato interessante perché la resistenza a piperacillina-tazobactam è rara mentre è confermata la piena sensibilità a moxifloxacina malgrado la recente descritta tendenza alla resistenza ai fluorochinoloni da parte di Bacteroides spp. L'accurata identificazione del batterio responsabile appare pertanto importante sia per la definizione della storia naturale e dei sintomi clinici della infezione sia per la verifica del pattern di sensibilità antibiotica. 